KYUNGPOOK Math. J. 51(2011), 339-344

http://dx.doi.org/10.5666/KMJ.2011.51.3.339

\title{
Kaplansky-type Theorems, II
}

\author{
GYu Whan CHANG $\sharp$ \\ Department of Mathematics, University of Incheon, Incheon 406-772, Korea \\ e-mail : whan@incheon.ac.kr \\ HWANKOO KIM*† \\ Department of Information Security, Hoseo University, Asan 336-795, Korea \\ e-mail : hkkim@hoseo.edu
}

Abstract. Let $D$ be an integral domain with quotient field $K, X$ be an indeterminate over $D$, and $D[X]$ be the polynomial ring over $D$. A prime ideal $Q$ of $D[X]$ is called an upper to zero in $D[X]$ if $Q=f K[X] \cap D[X]$ for some $f \in D[X]$. In this paper, we study integral domains $D$ such that every upper to zero in $D[X]$ contains a prime element (resp., a primary element, a $t$-invertible primary ideal, an invertible primary ideal).

\section{Introduction}

Let $D$ be an integral domain and $X$ be an indeterminate over $D$. It is well known that $D$ is a UFD if and only if every nonzero prime ideal of $D$ contains a nonzero prime element [12, Theorem 5]. This is the so-called Kaplansky's theorem. This type of theorems was studied by Anderson and Zafrullah [3] and Kim [13] to characterize GCD-domains, valuations domains, Prüfer domains, generalized GCD-domains, and PvMDs. (Definitions will be reviewed in the sequel.) In [5, Proposition 2.7], it is shown that $D[X]$ is a GWFD if and only if $D$ is a GWFD and each upper to zero in $D[X]$ contains a primary element. This work is motivated by the results ([12, Theorem 5], [3], [13], [5, Proposition 2.7]). The purpose of this paper is to study an integral domain $D$ such that each upper to zero in $D[X]$ contains a prime element (resp., a primary element, a $t$-invertible primary ideal, an invertible primary ideal). More precisely, we show that every upper to zero in $D[X]$ contains a prime element $f$ with $c(f)=D$ if and only if $D$ is a Bézout domain; every upper to zero in $D[X]$

\footnotetext{
* Corresponding Author.

Received January 18, 2011; accepted June 24, 2011.

2010 Mathematics Subject Classification: 13A15, 13F20, 13 F05.

Key words and phrases: Kaplansky theorem, upper to zero in $D[X]$, prime (primary) element.

$\#$ The first author's work was supported by the University of Incheon Research Fund in 2010.

$\dagger$ The second author's work was supported by Basic Science Research Program through the National Research Foundation of Korea(NRF) funded by the Ministry of Education, Science and Technology(2010-0011996).
} 
contains a primary element $f$ with $c(f)=D$ if and only if $D$ is a UMT-domain, each maximal ideal of $D$ is a $t$-ideal, and $C l(D[X])$ is torsion; and if $D$ is integrally closed, then every upper to zero in $D[X]$ contains an invertible (resp., $t$-invertible) primary ideal if and only if $D$ is an almost generalized GCD-domain (resp., $\mathrm{P} v \mathrm{MD}$ ).

We first introduce some definitions and notation. Let $D$ be an integral domain with quotient field $K, X$ an indeterminate over $D$, and $D[X]$ the polynomial ring over $D$. For any polynomial $f \in K[X]$, the content $c_{D}(f)$ (simply, $c(f)$ ) of $f$ is the fractional ideal of $D$ generated by the coefficients of $f$. An upper to zero in $D[X]$ is a prime ideal $Q_{f}=f K[X] \cap D[X]$ of $D[X]$, where $f \in D[X]$ is irreducible in $K[X]$. Let $I$ be a nonzero fractional ideal $I$ of $D$. Then $I^{-1}=\{x \in K \mid x I \subseteq D\}$, $I_{v}=\left(I^{-1}\right)^{-1}$, and $I_{t}=\bigcup\left\{J_{v} \mid J \subseteq I\right.$ is a nonzero finitely generated ideal $\}$. We say that $I$ is a $v$-ideal (resp., $t$-ideal) if $I=I_{v}$ (resp., $I=I_{t}$ ). A fractional ideal $I$ of $D$ is said to be $t$-invertible if $\left(I I^{-1}\right)_{t}=D$. A maximal $t$-ideal is an ideal of $D$ maximal among proper integral $t$-ideals of $D$. Let $t-\operatorname{Max}(D)$ be the set of maximal $t$-ideals. It is easy to see that if $D$ is not a field, then $t-\operatorname{Max}(D) \neq \emptyset$ and $D=\bigcap_{t-\operatorname{Max}(D)} D_{P}$.

An integral domain $D$ is a $U M T$-domain if every upper to zero in $D[X]$ is a maximal $t$-ideal; $D$ is a Prüfer $v$-multiplication domain $(\mathrm{P} v \mathrm{MD})$ if every nonzero finitely generated ideal of $D$ is $t$-invertible; $D$ is a $G C D$-domain if for any $0 \neq$ $a, b \in D, a D \cap b D$ (equivalently, $\left.(a, b)_{v}\right)$ is principal; $D$ is an almost $G C D$-domain (AGCD-doman) if for any $0 \neq a, b \in D$, there is a positive integer $n=n(a, b)$ such that $a^{n} D \cap b^{n} D$ is principal; $D$ is a generalized GCD-domain (GGCD-domain) if $a D \cap b D$ (equivalently, $(a, b)_{t}$ ) is invertible for any $0 \neq a, b \in D ; D$ is an almost $G G C D$-domain (AGGCD-domain) if for $0 \neq a, b \in D$, there is a positive integer $n=n(a, b)$ such that $a^{n} D \cap b^{n} D$ is invertible; and $D$ is a generalized weakly factorial domain (GWFD) if each nonzero prime ideal of $D$ contains a primary element (a nonzero nonunit $x \in D$ is primary if $x D$ is a primary ideal).

Let $T(D)$ be the group of $t$-invertible fractional $t$-ideals of $D$, and let $\operatorname{Prin}(D)$ be its subgroup of principal fractional ideals. Then the quotient group $C l(D)=$ $T(D) / \operatorname{Prin}(D)$ is an abelian group called the $(t-)$ class group of $D$. It is known that $D$ is a GCD-domain if and only if $D$ is a $\mathrm{P} v \mathrm{MD}$ and $C l(D)=0$ [6, Proposition 2]; if $D$ is integrally closed, then $D$ is an AGCD-domain if and only if $D$ is a $\mathrm{P} v \mathrm{MD}$ with $C l(D)$ torsion [15, Corollary 3.8]; and $D$ is an AGGCD-domain if and only if $D$ is an AGCD-domain with $C l(D)$ torsion [14, Theorem 5.1]. Any undefined terminology is standard, as in [8] or [12].

\section{Kaplansky-type theorems for uppers to zero}

Let $D$ be an integral domain with quotient field $K, D^{*}=D \backslash\{0\}, X$ be an indeterminate over $D$, and $D[X]$ be the polynomial ring over $D$.

Lemma 2.1(4, Lemma 2.1). If $f \in D[X] \backslash D$, then

(1) $f K[X] \cap D[X]=f D[X]$ if and only if $c(f)_{v}=D$;

(2) if $f$ is a product of primary elements in $D[X]$, then $f K[X] \cap D[X]=f D[X]$. 
It is well known that $D$ is a UFD if and only if every nonzero prime ideal of $D$ contains a nonzero prime element of $D[12$, Theorem 5].

Theorem 2.2. Every upper to zero in $D[X]$ contains a prime element if and only if $D$ is a GCD-domain.

Proof. $(\Rightarrow)$ For any $0 \neq a, b \in D$, let $f=a X+b$. Then $Q_{f}=f K[X] \cap D[X]$ is an upper to zero in $D[X]$, and so $Q_{f}$ contains a prime element $g$. Note that $\operatorname{ht}\left(Q_{f}\right)=1$; so $Q_{f}=g D[X]$, and hence $c(g)_{v}=D$ by Lemma 2.1 and $f=u g$ for some $u \in K$ (actually $u \in D$ ). Thus, $(a, b)_{v}=c(f)_{v}=u c(g)_{v}=u D$.

$(\Leftarrow)$ Suppose that $D$ is a GCD-domain, and let $h \in D[X]$ be such that $Q_{h}=$ $h K[X] \cap D[X]$ is an upper to zero in $D[X]$. Recall that a GCD-domain is integrally closed and $c(h)^{-1}$ is principal, say, $c(h)^{-1}=a D$. Thus, $a h$ is a prime element, because $Q_{h}=h c(h)^{-1}[X][8$, Corollary 34.9].

Corollary 2.3. Every upper to zero in $D[X]$ contains a prime element $f$ with $c(f)=D$ if and only if $D$ is a Bézout domain.

Proof. Let $a, b \in D$ be nonzero, and assume that $Q_{g}=g K[X] \cap D[X]$, where $g=a X+b$, contains a prime element $f$ with $c(f)=D$. Then $g=u f$ for some $u \in K$, and thus $(a, b)=c(g)=u D$, which means that $D$ is a Bézout domain. Conversely, assume that $D$ is a Bézout domain, and let $Q$ be an upper to zero in $D[X]$. Then $Q$ contains a prime element $f$ by Theorem 2.2, and since $D$ is a Bézout domain, $c(f)=a D$ for some $a \in D$. But, since $f$ is a prime element, $a D=D$, and thus $c(f)=D$.

Let $S$ be a multiplicative subset of $D$. We say that $S$ is an almost splitting (resp., almost $g^{d}$-splitting) set if, for each $0 \neq r \in D$, there is an integer $n=n(r) \geq 1$ such that $r^{n}=s t$ for some $s \in S$ and $t \in D$ with $\left(s^{\prime}, t\right)_{v}=D$ (resp., $\left(s^{\prime}, t\right)=D$ ) for all $s^{\prime} \in S$. Recall that $D$ is a quasi-AGCD-domain if $D^{*}$ is an almost splitting set in $D[X]$. The next theorem appears in [4, Theorem 2.4], which is a motivation for this paper.

Theorem 2.4. The following statements are equivalent.

(1) Every upper to zero in $D[X]$ contains a primary element.

(2) $D$ is a quasi-AGCD-domain.

(3) $D$ is a UMT-domain and $C l(D[X])$ is torsion.

Following [2], an integral domain $D$ is called an almost Bézout domain (ABdomain) if, for each $a, b \in D$, there is an integer $n \geq 1$ such that $\left(a^{n}, b^{n}\right)$ is principal. Obviously, if $D$ is integrally closed, then $D$ is an AB-domain if and only if $D$ is a Prüfer domain with $C l(D)$ torsion. It is known that $D$ is an AB-domain if and only if $D$ is an AGCD domain and each maximal ideal of $D$ is a $t$-ideal [2, Corollary 5.4]. So it is natural to call $D$ a quasi-AB-domain if $D$ is a quasi-AGCD-domain whose maximal ideals are $t$-ideals. Clearly, a quasi-AB-domain is a quasi-AGCD-domain, 
but not vice versa (for example, if $D$ is a GCD-domain, then $D[X]$ is a GCDdomain (hence a quasi-AGCD-domain) but not a quasi-AB-domain). However, if $D$ has (Krull) dimension one, then a quasi-AGCD-domain is a quasi-AB-domain.

Corollary 2.5. The following statements are equivalent.

(1) Every upper to zero in $D[X]$ contains a primary element $f$ with $c(f)=D$.

(2) $D$ is a UMT-domain, each maximal ideal of $D$ is a t-ideal, and $C l(D[X])$ is torsion.

(3) $D$ is a quasi-AB-domain.

Proof. (1) $\Rightarrow(2)$. By Theorem 2.4, $D$ is a UMT-domain and $C l(D[X])$ is torsion. Assume that there is a maximal ideal which is not a $t$-ideal. Then there is an $f \in D[X]$ such that $c(f)_{v}=D$ but $c(f) \subsetneq D$. Let $f=f_{1}^{e_{1}} \cdots f_{n}^{e_{n}}$ be the prime factorization of $f$ in $K[X]$ (note that $K[X]$ is a UFD). Then $f D[X]=f K[X] \cap$ $D[X]=\left(f_{1}^{e_{1}} K[X] \cap D[X]\right) \cap \cdots \cap\left(f_{n}^{e_{n}} K[X] \cap D[X]\right)$ by Lemma 2.1 and each $f_{i}^{e_{i}} K[X] \cap D[X]$ is a $Q_{i}$-primary ideal, where $Q_{i}=f_{i} K[X] \cap D[X](1 \leq i \leq n)$. Since each $Q_{i}$ is an upper to zero in $D[X], Q_{i}$ contains a primary element $g_{i}$ with $c\left(g_{i}\right)=D$. Clearly, each $g_{i}^{e_{i}} \in f_{i}^{e_{i}} K[X] \cap D[X]$, and so if we set $g:=g_{1}^{e_{1}} \cdots g_{n}^{e_{n}}$, then $g \in f D[X]$ and $c(g)=D$. Thus, $c(f)=D$, a contradiction.

$(2) \Rightarrow(1)$. Let $Q$ be an upper to zero in $D[X]$. Since $D$ is a UMT-domain, $Q$ is $t$-invertible. Also, since $C l(D[X])$ is torsion, there is an integer $n \geq 1$ such that $\left(Q^{n}\right)_{t}=f D[X]$ for some $f \in D[X]$. Note that $f$ is primary, and since $Q$ is a maximal $t$-ideal, $c(f)_{t}=D$. Thus, $f$ is a primary element with $c(f)=D$, because each maximal ideal is a $t$-ideal.

$(2) \Leftrightarrow(3)$. This follows from Theorem 2.4.

It is naturally asked that it follows from the definition that if $D$ is a quasi-ABdomain, then $D^{*}$ is an almost $g^{d}$-splitting set in $D[X]$. However, $(a, X) \neq D[X]$ for any nonunit $a \in D$. Hence $D^{*}$ cannot be an almost $g^{d}$-splitting set in $D[X]$.

Corollary 2.6. The following statements are equivalent for an integrally closed domain $D$.

(1) Every upper to zero in $D[X]$ contains a primary element $f$ with $c(f)=D$.

(2) $D$ is a Prüfer domain and $C l(D)$ is torsion.

(3) $D$ is a quasi-AB-domain.

(4) $D$ is an AB-domain.

Proof. (1) $\Leftrightarrow(2)$. Note that an integrally closed domain is a Prüfer domain if and only if it is a UMT-domain whose maximal ideals are $t$-ideals. Also, if $D$ is integrally closed, then $C l(D[X])=C l(D)([7$, Theorem 3.6]). Thus, the result follows from Corollary 2.5.

$(1) \Leftrightarrow(3)$. This follows from Corollary 2.5 .

(2) $\Leftrightarrow(4)$. This is clear. 
Corollary 2.7. If $D$ is a quasi-AB-domain, then each overring $R$ of $D$ is a quasi$A B$-domain. In particular, if $R$ is integrally closed, then $R$ is a Prüfer domain with torsion class group.

Proof. Let $Q$ be an upper to zero in $R[X]$. Then there is an $f \in K[X]$ such that $Q=f K[X] \cap R[X]$, and hence $Q \cap D[X]=f K[X] \cap D[X]$ is an upper to zero in $D[X]$. By Corollary 2.5, there is a primary element $g \in Q \cap D[X]$ such that $c_{D}(g)=D$. Clearly, $g \in Q$ and $c_{R}(g)=R$; in particular, $Q$ is a maximal $t$-ideal of $R[X][9$, Theorem 1.4]. Note that, since $g$ is a primary element of $D[X]$, there exist some $u \in K$ and an integer $n \geq 1$ such that $g=u f^{n}$. Hence $\sqrt{g R[X]}=f K[X] \cap R[X]$, and thus $g$ is a primary element of $R[X]$ [5, Lemma 2.1]. Thus, $R$ is a quasi-ABdomain by Corollary 2.5. In particular, if $R$ is integrally closed, then $R$ is a Prüfer domain with torsion class group by Corollary 2.6.

It is well known that if $D$ is integrally closed, then $D$ is a UMT-domain if and only if $D$ is a $\mathrm{P} v \mathrm{MD}$ [9, Proposition 3.2]. Also, it is known that $D$ is a Krull domain if and only if every nonzero prime $(t$-)ideal contains a $t$-invertible prime ideal $[11$, Theorem3.6] and $D$ is a GGCD-domain if and only if each upper to zero in $D[X]$ is invertible [1, Theorem 15].

Theorem 2.8. If $D$ is integrally closed, then

(1) every upper to zero in $D[X]$ contains a t-invertible primary ideal if and only if $D$ is a PvMD;

(2) every upper to zero in $D[X]$ contains an invertible primary ideal if and only if $D$ is an almost generalized GCD-domain.

Proof. (1) $(\Rightarrow)$ Let $Q$ be an upper to zero in $D[X]$, and let $I$ be a $t$-invertible primary $t$-ideal contained in $Q$. Since $\operatorname{ht}(Q)=1$, we have $\sqrt{I}=Q$. Let $N_{v}=\{f \in$ $\left.D[X] \mid c(f)_{v}=D\right\}$, and suppose $Q \cap N_{v}=\emptyset$. Then $I_{N_{v}} \subseteq Q_{N_{v}} \subsetneq D[X]_{N_{v}}$. Since $I$ is $t$-invertible, $I_{N_{v}}$ is invertible (cf. [10, Proposition 2.1(3)]), and hence $I_{N_{v}}$ is principal [10, Theorem 2.14]. So $Q_{N_{v}}=\sqrt{I_{N_{v}}}$ is a maximal $t$-ideal [5, Lemma 2.1]. This is contrary to the fact that $\operatorname{Max}\left(D[X]_{N_{v}}\right)=t-\operatorname{Max}\left(D[X]_{N_{v}}\right)=\left\{P[X]_{N_{v}} \mid P \in t\right.$ $\operatorname{Max}(D)\}\left[10\right.$, Propositions 2.1 and 2.2]. So $Q \cap N_{v} \neq \emptyset$, and thus $Q$ is a maximal $t$-ideal [9, Theorem 1.4]. Thus, $D$ is a $\mathrm{P} v \mathrm{MD}$.

$(\Leftarrow)$ Let $Q$ be an upper to zero in $D[X]$. Then $Q$ is a maximal $t$-ideal, because a $\mathrm{P} v \mathrm{MD}$ is a UMT-domain. Thus, $Q$ is a $t$-invertible prime (hence primary) $t$-ideal [9, Proposition 1.4].

$(2)(\Rightarrow)$ We first note that $D$ is a $\mathrm{P} v \mathrm{MD}$ by (1). Let $0 \neq a, b \in D$, and put $f=a X+b$. Then $Q_{f}=f K[X] \cap D[X]$ is an upper to zero in $D[X]$, and so $Q_{f}$ contains an invertible primary ideal $A$. It is easy to see that $Q_{f}=f c(f)^{-1}[X][8$, Corollary 34.9] and $A=\left(\left(Q_{f}\right)^{n}\right)_{t}$ for some positive integer $n$. Note that $\left(\left(Q_{f}\right)^{n}\right)_{t}=$ $f^{n} c\left(f^{n}\right)^{-1}[X]$ and $c\left(f^{n}\right)^{-1}=\left(c(f)^{n}\right)^{-1}=\left((a, b)^{n}\right)^{-1}$. Thus, $\left(a^{n}, b^{n}\right)_{t}$ is invertible, because $(a, b)_{t}$ is $t$-invertible by $(1)$, and so $\left(\left((a, b)^{n}\right)^{-1}\right)^{-1}=\left((a, b)^{n}\right)_{t}=\left(a^{n}, b^{n}\right)_{t}$ [2, Lemma 3.3]. 
$(\Leftarrow)$ Let $Q_{g}=g K[X] \cap D[X]$, where $g \in D[X]$, be an upper to zero in $D[X]$. Note that $Q_{g}=g c(g)^{-1}[X][8$, Corollary 34.9], because $D$ is integrally closed. Note also that, since $D$ is an almost GGCD-domain, there is a positive integer $m$ such that $\left(c(g)^{m}\right)_{t}=c\left(g^{m}\right)_{t}$ is invertible by (1), [8, Proposition 34.8], and [14, Theorem 3.2]. Thus $\left(Q_{g}^{m}\right)_{t}=g^{m} K[X] \cap D[X]=g^{m} c\left(g^{m}\right)^{-1}[X]$ is an invertible primary ideal. $\square$

Acknowledgement. The authors would like to thank the referee for his/her useful comments.

\section{References}

[1] D. D. Anderson, T. Dumitrescu, and M. Zafrullah, Quasi-Schreier domains, II, Comm. Algebra, 35(2007), 2096-2104.

[2] D. D. Anderson and M. Zafrullah, Almost Bézout domains, J. Algebra, 142(1991), 285-309.

[3] D. D. Anderson and M. Zafrullah, On a theorem of Kaplansky, Boll. Un. Mat. Ital. A (7), 8(1994), 397-402.

[4] D. F. Anderson and G. W. Chang, Almost splitting sets in integral domains II, J. Pure Appl. Algebra, 208(2007), 351-359.

[5] D. F. Anderson, G. W. Chang, and J. Park, Generalized weakly factorial domains, Houston J. Math., 29(2003), 1-13.

[6] A. Bouvier, Le groupe des classes d'un anneau intègre, 107ème Congres des Sociétés Savantes, Brest, 1982, fasc. IV, 85-92.

[7] S. Gabelli, On divisorial ideals in polynomial rings over Mori domains, Comm. Algebra, 15(1987), 2349-2370.

[8] R. Gilmer, Multiplicative Ideal Theory, Marcel Dekker, New York, 1972.

[9] E. Houston and M. Zafrullah, On t-invertibility, II, Comm. Algebra, 17(1989), 19551969.

[10] B. G. Kang, Prüfer v-multiplication domains and the ring $R[X]_{N_{v}}$, J. Algebra, 123(1989), 151-170.

[11] B. G. Kang, On the converse of a well-known fact about Krull domains, J. Algebra, 124(1989), 284-299.

[12] I. Kaplansky, Commutative Rings, rev. ed., Univ. of Chicago, Chicago, 1974.

[13] H. Kim, Kaplansky-type theorems, Kyungpook Math. J., 40(2000), 9-16.

[14] R. Lewin, Almost generalized GCD-domains, Lecture Notes in Pure and Appl. Math., Marcel Dekker, 189(1997), 371-382.

[15] M. Zafrullah, A general theory of almost factoriality, Manuscripta Math., 51(1985), 29-62. 